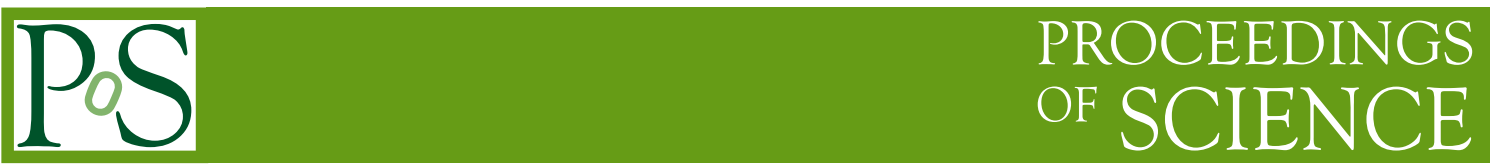

\title{
Standard Model Higgs Results from ATLAS and CMS
}

\section{Johannes Elmsheuser*, on behalf of the ATLAS and CMS Collaborations Brookhaven National Laboratory, USA}

E-mail: johannes.elmsheuserecern.ch

This paper presents an overview of the current status of Higgs boson measurements from the ATLAS and CMS experiments at the LHC. The Higgs boson mass and coupling analyses using proton-proton collision data at the center-of-mass energies of $7 \mathrm{TeV}$ and $8 \mathrm{TeV}$ will be discussed. Also preliminary Higgs boson cross-section measurements at the center-of-mass energy of 13 $\mathrm{TeV}$ are presented.

Prospects for Charged Higgs Discovery at Colliders 3-6 October 2016

Uppsala, Sweden

\footnotetext{
* Speaker.
} 


\section{Introduction}

The Standard Model (SM) of particle physics describes exceptionally well nearly all of experimental particle physics data. In the SM, the Brout-Englert-Higgs (BEH) mechanism $[1,2,3]$ spontaneously breaks electroweak (EW) gauge symmetry and generates mass terms for the $W$ and $Z$ gauge bosons and also for the fermion fields via Yukawa couplings. As a consequence of the BEH mechanism, the SM predicts the existence of a heavy scalar particle (the Higgs boson, $H$ ); the search for this particle is a highlight of the Large Hadron Collider (LHC) physics program.

In the summer of 2012 the ATLAS [4] and CMS [5] collaborations discovered a new particle with a mass of approximately $125 \mathrm{GeV}[6,7]$ via decays to photon, $W$ and $Z$ boson pairs with rates consistent with those of the SM Higgs boson. Subsequent measurements of the properties of this particle are all consistent with the SM Higgs boson interpretation. In the following sections the most recent results from studies of this Higgs boson using data collected by the ATLAS and CMS experiments are presented. First, a brief summary of the results with the full Run 1 dataset recorded in 2011 and 2012 at center-of-mass energies of 7 and $8 \mathrm{TeV}$, respectively, corresponding to about $25 \mathrm{fb}^{-1}$ per experiment is given. Then results using a partial Run 2 dataset recorded in 2015 and 2016 at a center-of-mass energy of $13 \mathrm{TeV}$ corresponding to up about $15 \mathrm{fb}^{-1}$ per experiment are presented.

\section{Higgs boson results in Run 1}

The dominant Higgs boson production modes are the gluon-gluon fusion ( $\mathrm{ggF}$ ), vector boson fusion (VBF), the production in association with a vector boson $(\mathrm{VH}, \mathrm{V}=W$ or $Z$ ) and in association with top quarks $(t t H)$. The most sensitive Higgs boson decay channels at the LHC are the $\gamma \gamma, Z Z$, $W W, \tau \tau$ and $b b$ decay modes.

The Higgs boson mass $m_{H}$ has been measured independently by the ATLAS and CMS experiments using the Run 1 dataset in several decay channels. Channels with the best mass resolution $(H \rightarrow \gamma \gamma$ and $H \rightarrow Z Z \rightarrow 4 \ell(\ell=e, \mu))$ are used in the combination of analyses from the two experiments [8]. The Higgs boson mass based on this combination is $m_{H}=125.09 \pm$ 0.21 (stat.) \pm 0.11 (syst) $\mathrm{GeV}$. The measurement is dominated by the statistical uncertainty. The systematic uncertainty is dominated by the uncertainties of the photon, electron, muon energy and momentum scales and resolutions. The results of the Higgs boson mass measurements performed by the individual experiments as well as the combined values are shown in Fig. 1 (left).

The combined ATLAS and CMS measurements of the Higgs boson production and decay rates, as well as constraints on its couplings to vector bosons and fermions have also been determined [9]. The combination is based on the previously mentioned production processes and and decay channels plus the $H \rightarrow \mu \mu$ channel. All results assume a value of $m_{H}=125.09 \mathrm{GeV}$. The combined signal yield relative to the Standard Model prediction is measured to be $1.09 \pm 0.11$. The combined measurements lead to observed (expected) significances for the vector boson fusion production process and for the $\tau \tau$ decay channel of 5.4 (4.6) and 5.5 (5.0) standard deviations, respectively. The data are consistent with the Standard Model predictions for all measurements. Fig. 1 (right) shows the best fit values of $\sigma \cdot B$ for each specific channel as obtained from the generic parameterisation for the combination of the ATLAS and CMS measurements. 

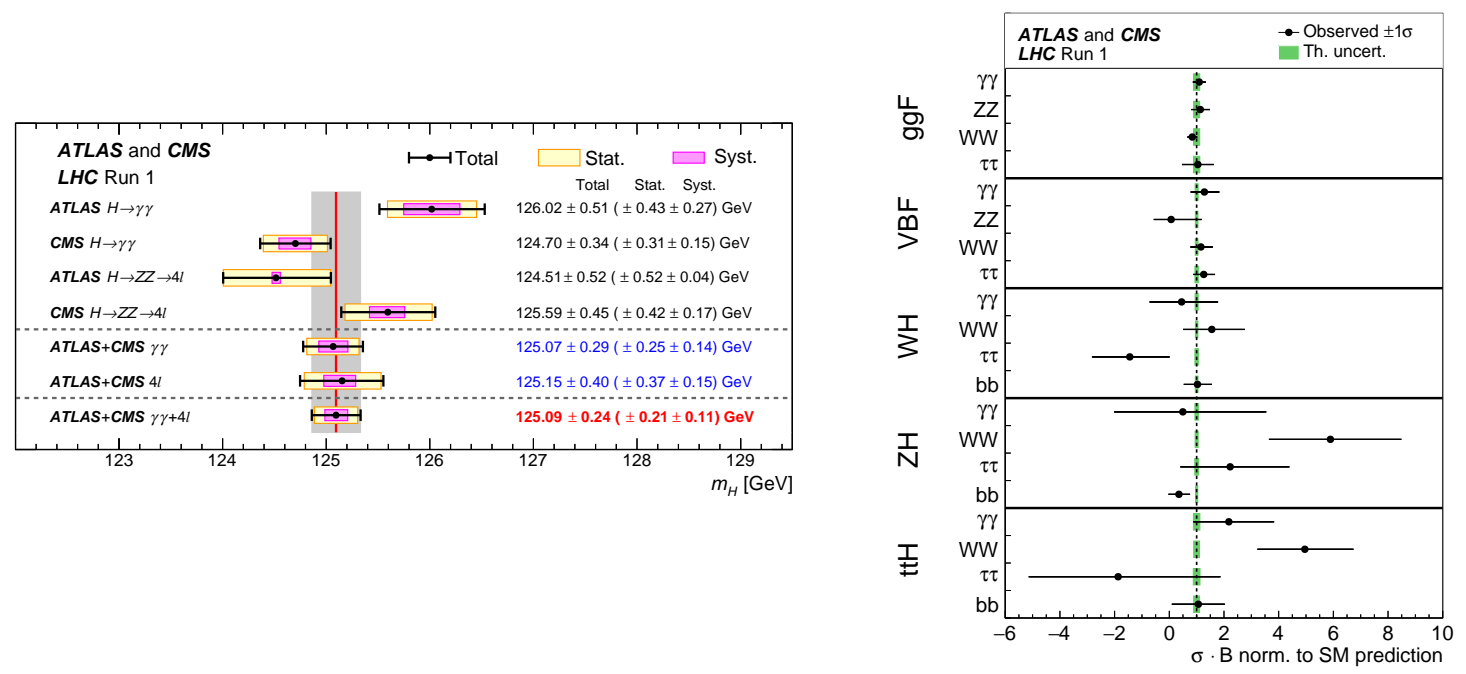

Figure 1: Left: Summary of Higgs boson mass measurements from the individual analyses of ATLAS and CMS and from the combined analysis. The systematic (narrower, magenta-shaded bands), statistical (wider, yellow-shaded bands), and total (black error bars) uncertainties are indicated [8]. Right: Best fit values of $\sigma \cdot B$ for each specific combination, as obtained from the generic parameterisation for the combination of the ATLAS and CMS measurements. The error bars indicate the $1 \sigma$ intervals. The fit results are normalised to the SM predictions for the various parameters and the shaded bands indicate the theoretical uncertainties in these predictions [9]. For both analysis the data correspond to integrated luminosities per experiment of approximately $5 \mathrm{fb}^{-1}$ at $\sqrt{s}=7 \mathrm{TeV}$ (recorded in 2011) and $20 \mathrm{fb}^{-1}$ at $\sqrt{s}=8 \mathrm{TeV}$ (recorded in 2012).

\section{Higgs boson results in Run 2}

\section{1 $H \rightarrow \gamma \gamma$}

The $H \rightarrow \gamma \gamma$ channel provides relatively high event yields with a good mass resolution $\left(m_{\gamma \gamma}\right)$ but has a low signal-over-background ratio. In the analyses two isolated photons are selected in various categories targeted at the different production modes [10,11]. For the VBF category two additional jets with a large rapidity gap, for the $V H$ category additional leptons or missing transverse energy and for the $t H$ category event topologies consistent with additional top quarks in the event are required. The signal is extracted from a fit in the invariant mass distribution of the two photons $m_{\gamma \gamma}$ as shown in Fig. 2 (left). The background consists mainly of events from continuum $\gamma \gamma, \gamma+$ jet and di-jet production and the composition is measured using a sideband method. The dominant systematic uncertainties are the photon energy scale and resolution. The signal strengths are determined to be $\mu=0.85_{-0.20}^{+0.22}$ (ATLAS) and $\mu=0.91 \pm 0.20$ (CMS) for $m_{H}=125.09 \mathrm{GeV}$. Fiducial cross sections in which event yields are corrected for smaller detector inefficiencies and resolution for a minimal theoretical modelling are extracted; $\sigma_{\text {fid }}=43.2 \pm 14.9$ (stat) \pm 4.9 (syst) pb (SM: 62.8 $8_{-4.4}^{+3.4} \mathrm{pb}$ ) for ATLAS and $\sigma_{\text {fid }}=69_{-22}^{+16}$ (stat) ${ }_{-6}^{+8}$ (syst) pb (SM: $73.8 \pm 3.8 \mathrm{pb}$ ) for CMS. The values differ due to the different acceptance in ATLAS and CMS. The differential cross section as a function of the diphoton transverse momentum, the absolute rapidity of the diphoton system and the jet multiplicity are measured and compared to the SM prediction. Fig. 2 (right) shows this measurement for the diphoton transverse momentum in ATLAS. 

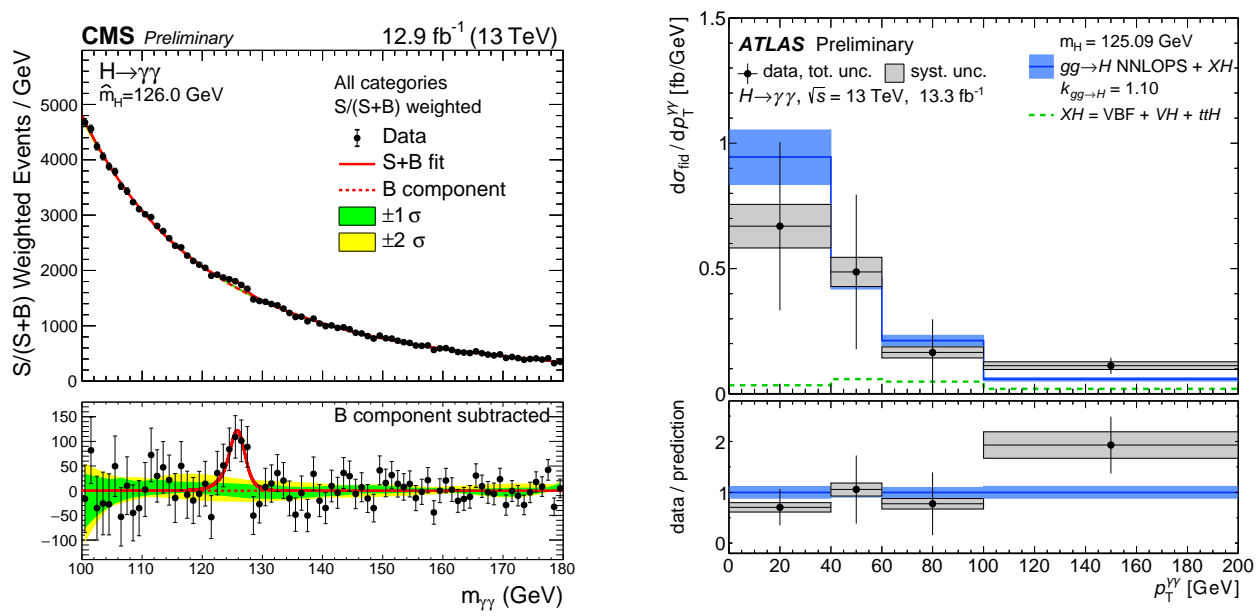

Figure 2: Left: $H \rightarrow \gamma \gamma$, data points (black) and signal plus background model fits for the categories are summed weighted by their sensitivity for the CMS analysis with an integrated luminosity of $12.9 \mathrm{fb}^{-1}$. The bottom plot shows the residuals after background subtraction [11]. Right: The differential cross section for $p p \rightarrow H \rightarrow \gamma \gamma$ as a function of the diphoton transverse momentum for the ATLAS analysis with an integrated luminosity of $13.3 \mathrm{fb}^{-1}[10]$.

\section{2 $H \rightarrow Z Z$}

The analyses in the $H \rightarrow Z Z \rightarrow 4 \ell(\ell=e, \mu)$ decay channel require 2 pairs of same flavour opposite charged isolated leptons where one pair has a dilepton invariant mass close to the $Z$ boson mass $[12,13]$. The channel has a low event rate but a very high signal-to-background ratio with a narrow signal peak on a flat background. The background mainly consists of event from $Z Z^{*}$ continuum production. The signal is extracted from a fit in the invariant $4 \ell$ mass distribution as shown in Fig. 3 (left). The dominant systematic uncertainties are from luminosity and lepton scale factor uncertainties. The fiducial cross sections are measured to be $\sigma_{\text {fid }}=4.54_{-0.90}^{+1.02} \mathrm{pb}$ (SM: $3.07_{-0.25}^{+0.21}$ $\mathrm{pb}$ ) for ATLAS and $\sigma_{\text {fid }}=2.29_{-0.64}^{+0.74}(\mathrm{stat})_{-0.23}^{+0.30}$ (syst) pb (SM: $2.53 \pm 0.13 \mathrm{pb}$ ) for CMS. The differential cross section as a function of the transverse momentum $p_{\mathrm{T}}(H)$ and the jet multiplicity are measured and compared to the SM prediction. Fig. 3 (right) shows this measurement for $p_{\mathrm{T}}(H)$ in CMS.

\section{3 $H \rightarrow \gamma \gamma$ and $H \rightarrow Z Z$ combination}

A combined measurement based on the analyses of the $H \rightarrow \gamma \gamma$ and $H \rightarrow Z Z \rightarrow 4 \ell$ decay channels in ATLAS yields a global signal strength $\mu=1.13_{-0.17}^{+0.18}$ for a Higgs boson mass of 125.09 $\mathrm{GeV}$ [14]. The cross section of $p p \rightarrow H+X$ in the full phase space is determined from fiducial cross section measurements to be $59.0_{-9.2}^{+9.7}$ (stat) ${ }_{-3.5}^{+4.4}$ (syst) pb with a Standard Model prediction of $55.5_{-3.4}^{+2.4} \mathrm{pb}$. Fig. 4 (left) shows the contours for $\sigma \cdot B$ in the ggF and VBF production for both channels whereas Fig. 4 (right) shows the fiducial cross section dependence on the center-ofmass energy. In the measurement of the total cross section, instead of attempting to separate the different Higgs boson production processes or using the categorisation to enhance the sensitivity, the inclusive event samples are used to minimise the model dependence. 

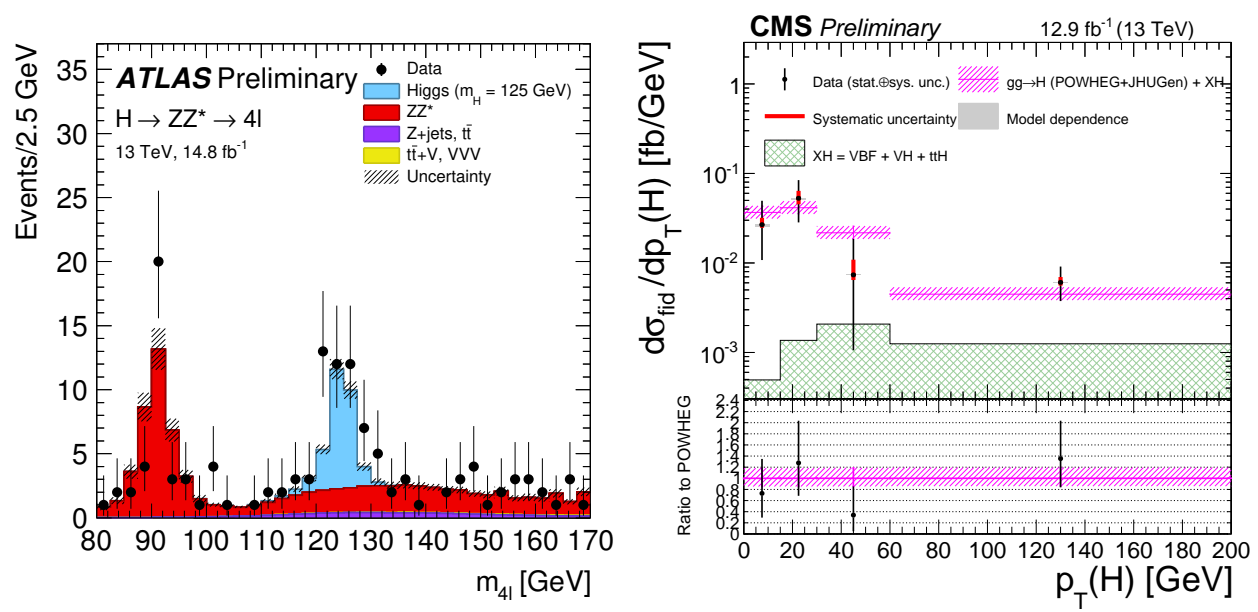

Figure 3: Left: $H \rightarrow Z Z$, the $m_{4 \ell}$ distribution of the selected candidates, compared to the background expectation for the ATLAS analysis with an integrated luminosity of $14.8 \mathrm{fb}^{-1}$ [12]. Right: Result of the differential cross section measurement for $p_{\mathrm{T}}(H)$ for the CMS analysis with an integrated luminosity of 12.9 $\mathrm{fb}^{-1}[13]$.
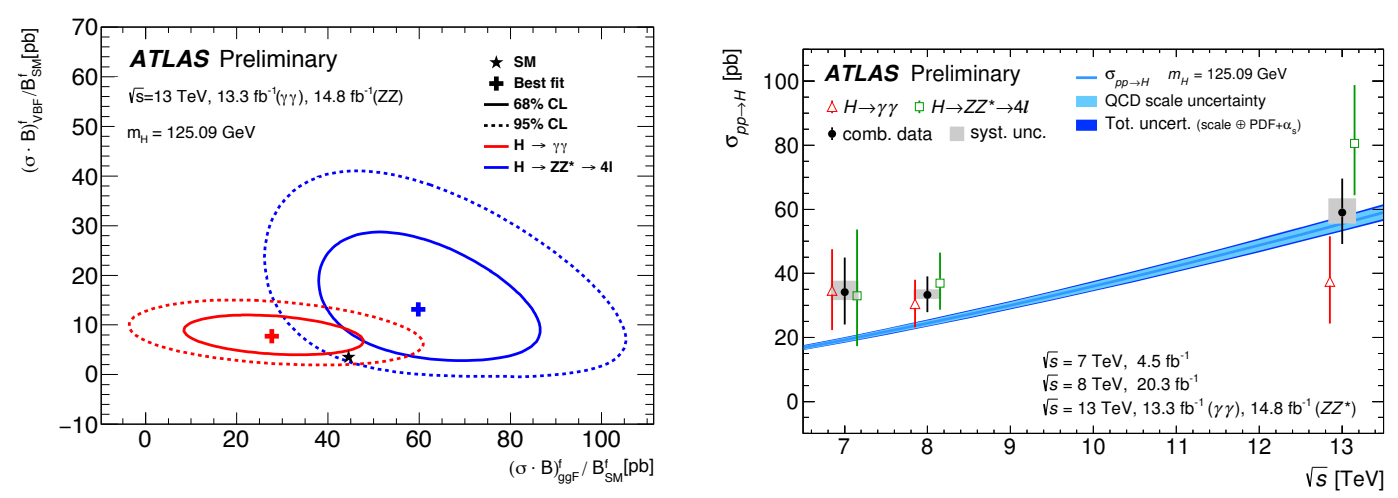

Figure 4: Left: Contours in the $(\sigma B)_{g g F}^{f} / B_{S M}^{f}$ vs. $(\sigma B)_{V B F}^{f} / B_{S M}^{f}$ plane as measured in $H \rightarrow \gamma \gamma$ and $H \rightarrow Z Z$, together with the SM prediction in ATLAS with an integrated luminosity of $13.3 \mathrm{fb}^{-1}$ [14]. Right: Total $p p \rightarrow H+X$ cross sections measured at different center-of-mass energies compared to SM predictions at up to N3LO in QCD in ATLAS with an integrated luminosity of $14.8 \mathrm{fb}^{-1}$ in Run 2 [14].

\section{4 $t t H$ production}

Using Run 1 data, the coupling of the Higgs boson to the top quark has been determined indirectly mainly from the top quark contribution to the gluon fusion and di-photon decay loops [9]. Higgs boson production in association with a pair of top quarks, $t t H$, is the most promising channel for observing such a direct coupling. The search for $t t H$ production is carried out in the $b b, W W$ (multilepton) and $\gamma \gamma$ decay channels of the Higgs boson by ATLAS and CMS $[15,16,17,18]$. In the $b b$ decay channel, events are categorised based on the number of leptons and b-jets. The main backgrounds are due to $t t+$ heavy flavour production and the dominant systematic uncertainties arise from the uncertainties of the signal and background modelling and normalisation which are larger than the statistical uncertainties. Since there is a rather small signal to background ratio mul- 
tivariate analysis techniques using Boosted Decision Trees are applied to improve the sensitivity and extract the signal. The signal strengths are determined to be $\mu=2.1_{-0.9}^{+1.0}$ (ATLAS, $13.2 \mathrm{fb}^{-1}$ ) and $\mu=2.0 \pm 1.8\left(\mathrm{CMS}, 2.7 \mathrm{fb}^{-1}\right)$ for $m_{H}=125.09 \mathrm{GeV}$. In the multilepton channel the signal signature consists of two to four charged leptons, two or more jets and one or more b-tagged jets. The dominant systematic uncertainty is due to uncertainty of the determination of fake lepton identification and non-prompt background. The signal strengths in this production mode are determined to be $\mu=2.5_{-1.1}^{+1.3}$ (ATLAS, $13.2 \mathrm{fb}^{-1}$ ) and $\mu=2.0_{-0.7}^{+0.8}\left(\mathrm{CMS}, 12.9 \mathrm{fb}^{-1}\right.$ ) for $m_{H}=125.09 \mathrm{GeV}$.

A combination of the searches for Higgs boson production in association with top quarks $(t t H)$ in the $\gamma \gamma$, multilepton, and $b b$ decay channels in ATLAS yields a signal strength $\mu=1.8 \pm 0.7$ [19]. This corresponds to an observed significance of $2.8 \sigma$, where $1.8 \sigma$ would be expected in the presence of Standard Model $t t H$ production. Fig. 5 (left) shows the summary of the different $t t H$ signal strength measurements in ATLAS.

\section{$3.5 t H \rightarrow b b$ production}

The Higgs boson production in association with a single top quark has a very small production cross section compared to the so far discussed production modes. A direct search for the production of a Higgs boson in association with a single top quark either via the $t$ channel or via the associated production with a $\mathrm{W}$ boson and using Higgs boson decays to a bottom quark-antiquark pair and semileptonic top quark decays is performed using CMS data [20]. The signal is selected in events with electron or muon decays of the $W$ boson from the top quark decay and $4 \mathrm{~b}$-tagged jets. The overwhelming background is reduced using a Boosted Decision Tree whose output distribution is used to extract the signal yield as shown in Fig. 5 (right). The expected (observed) upper limits are 113.7. $\sigma_{S M}\left(98.6 \cdot \sigma_{S M}\right)$. The observed (expected) upper limit for an inverted top coupling scenario with a negative Yukawa coupling of the top quark is 6.0 (6.4) times the predicted cross section.
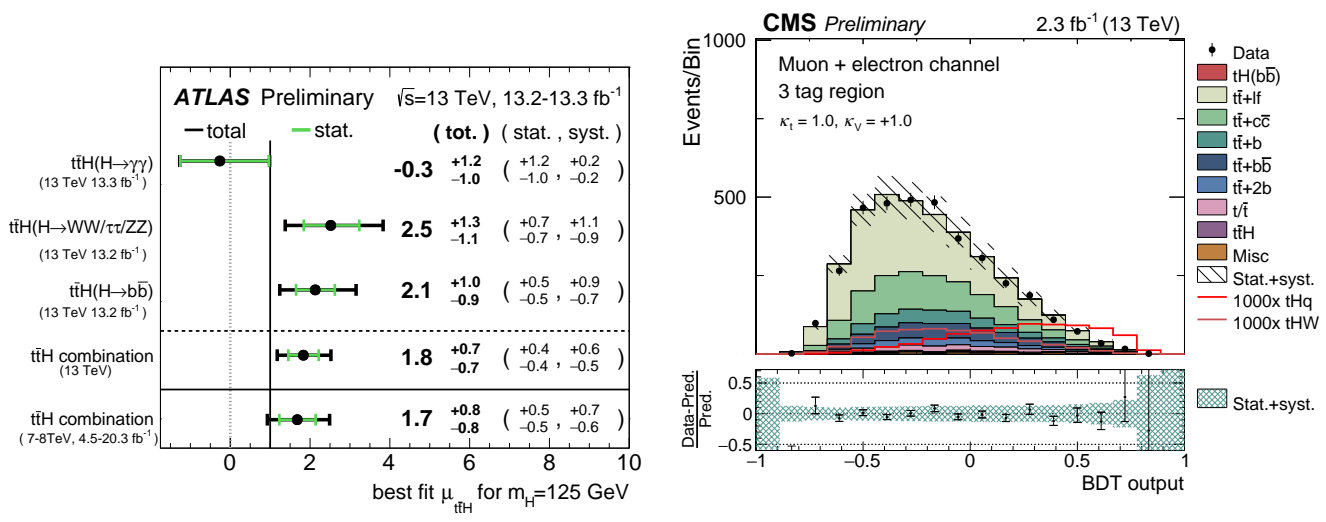

Figure 5: Left: Summary of the observed $t t H$ signal strength measurements from the individual analyses and for their combination, assuming $m_{H}=125 \mathrm{GeV}$ for ATLAS with an integrated luminosity of up to 13.3 $\mathrm{fb}^{-1}$ [19]. Right: Distribution of the classification BDT response for the $t H \rightarrow b b$ production search in CMS with an integrated luminosity of $2.3 \mathrm{fb}^{-1}$. The signal distributions correspond to the expected contributions scaled by the factors given in the legends [20]. 


\section{6 $H \rightarrow b b$ production}

The $b b$ decay channel is extremely challenging at the LHC, despite its high expected branching ratio at $m_{H}=125 \mathrm{GeV}$. The ggF production is not accessible as the signal cannot be separated from the overwhelming non-resonant $b b$ background. For the VBF production in this decay channel the signal to background ratio is only marginally better. Therefore the main search channel are the $V H$ production, which uses an additional lepton from $W$ or $Z$ decays $(Z \rightarrow v v, W \rightarrow \ell v, Z \rightarrow \ell \ell)$ [21]. Boosted Decision Trees are used to improve the signal to background ratio. The dominant backgrounds are $Z+b$-jets and $t t$ production and the dominant systematic uncertainties arise from the uncertainties on the b-tagging efficiency and the background normalisation. The signal is extracted from the invariant mass $m_{b b}$ which is shown in Fig. 6 (left) for the one-lepton channel. The combination of all the channel yields a signal strength $\mu=0.21_{-0.35}^{+0.36}$ (stat.) \pm 0.36 (syst.). This corresponds to an observed significance of 0.42 standard deviations compared with an expected sensitivity of 1.94 .

Similarly, analyses in the VBF production mode which has a larger production cross section compared to the $V H$ production have been carried out [22, 23]. In addition to the previously described analysis the rapidity gap of the two jets from the VBF production is used (see Fig. 6 (right)). In the ATLAS analysis, an additional photon from initial or final state radiation is required to further suppress backgrounds from multi-jet production. The upper observed (expected) limits are $4.0(6.0) \cdot \sigma_{S M}(\mathrm{ATLAS})$ and $3.4(2.3) \cdot \sigma_{S M}(\mathrm{CMS})$.
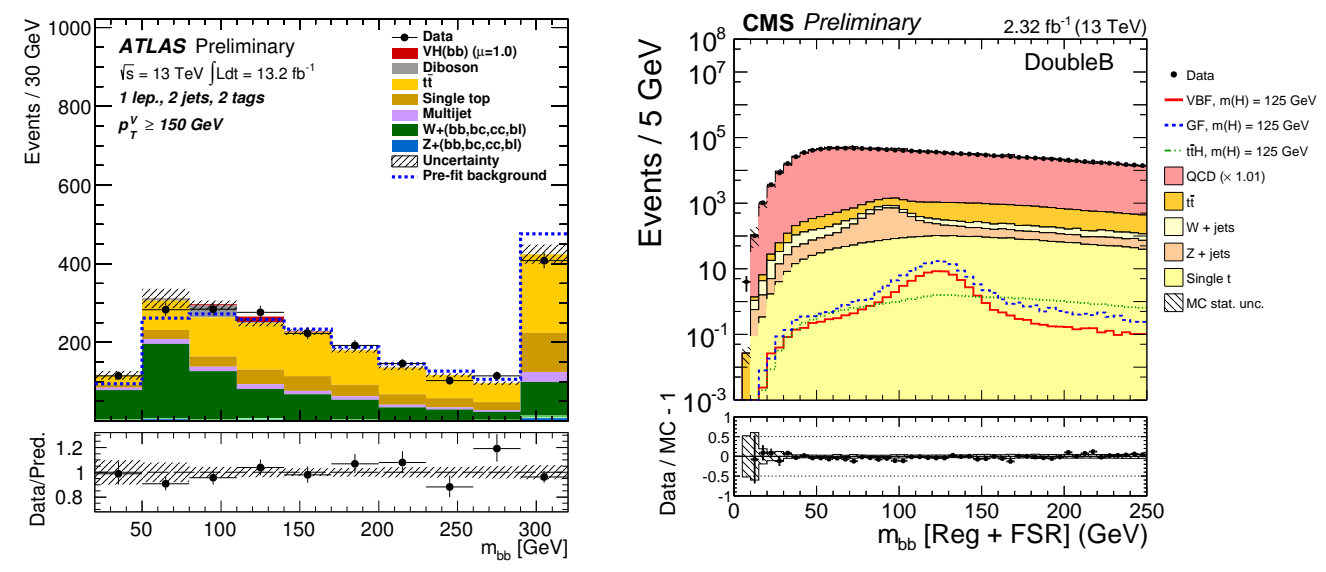

Figure 6: Left: The $m_{b b}$ post-fit distributions in the 1-lepton channel for 2-jet, 2 b-tag events in the $H \rightarrow b b$ analysis in ATLAS with an integrated luminosity of $13.2 \mathrm{fb}^{-1}$ [21]. Right: The invariant mass distribution of the two b-jet candidates, after a jet $p_{\mathrm{T}}$ regression to improve the $b b$ mass resolution, for events with more than one b-tagged jet with an integrated luminosity of $2.3 \mathrm{fb}^{-1}[23]$.

\section{7 $H \rightarrow \mu \mu$}

The $H \rightarrow \mu \mu$ decay has a clean final-state signature and is the only channel where the Higgs coupling to second generation fermions can be measured at the LHC [24]. This is a very challenging measurement due to the small $H \rightarrow \mu \mu$ branching ratio and high SM backgrounds. The dominant irreducible SM background is the $Z / \gamma^{*} \rightarrow \mu \mu$ process with a very high production rate compared to an expected Higgs signal. The signal is extracted from a fit to the invariant mass $m_{\mu \mu}$ 
distribution after the selection of 2 isolated muons (see Fig. 7 (left)). The observed (expected) upper limit using the Run 1 data is $7.1(7.2) \cdot \sigma_{S M}$ and for the analysed Run 2 dataset it is $4.4(5.5) \cdot \sigma_{S M}$. For the combination of both datasets the observed (expected) upper limit is $3.5(4.5) \cdot \sigma_{S M}$. The observed (expected) upper limit using Run 1 data in CMS is $7.4\left(6.5_{-1.9}^{+2.8}\right)$ times the SM value [25].
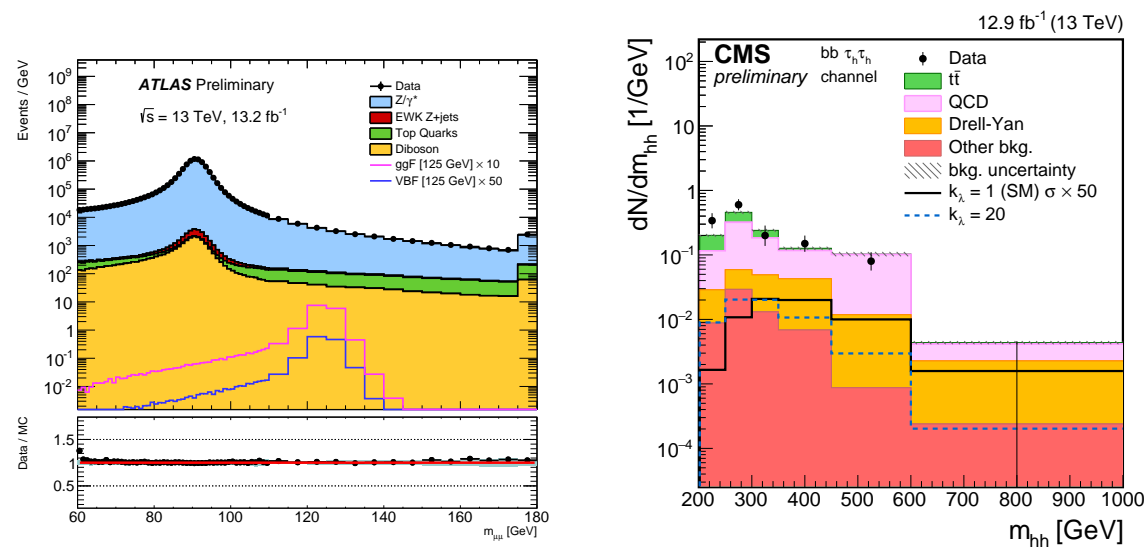

Figure 7: Left: The $m_{\mu \mu}$ in the $H \rightarrow \mu \mu$ analysis in ATLAS with an integrated luminosity of $13.2 \mathrm{fb}^{-1}$ [24]. Right: Distribution of the reconstructed visible four-body mass $\left(m_{h h}\right)$ after applying the event selection for the $b b \tau_{h} \tau_{h}$ channel in CMS with an integrated luminosity of $12.9 \mathrm{fb}^{-1}$ [27]. The black unshaded histogram is the signal expectation for the SM scaled by a factor 50 .

\subsection{Di-Higgs production}

Several analyses are carried out to search for di-Higgs production. Given the current collected integrated luminosity by ATLAS and CMS there is no sensitivity to di-Higgs production in the SM due to the small cross section. Many models of new physics predict cross sections for Higgs boson pair production that are significantly greater than the SM prediction. Two example searches are briefly discussed and the results for the non-resonant di-Higgs production are quoted here: $h h \rightarrow b \bar{b} b \bar{b}$ in ATLAS [26] and $h h \rightarrow b \bar{b} \tau^{+} \tau^{-}$in CMS [27]. The first analysis selects 4 b-tagged jets and the signal is extracted from the $m_{4 j}$ distribution. The dominant systematic uncertainties arise from the uncertainties on modelling of the background and the b-tagging. The observed upper limit on $h h \rightarrow b \bar{b} b \bar{b}$ production is $330 \mathrm{fb}$ with a SM prediction of $11.3 \pm 0.9 \mathrm{fb}$.

In the $h h \rightarrow b \bar{b} \tau^{+} \tau^{-}$analysis events with two b-tagged jets are selected together with two $\tau$ in their different decay channels (see Fig. 7 (right) for the $b b \tau_{h} \tau_{h}$ channel). The dominant systematic uncertainty is due to the uncertainty of the background modelling which stems from $t t$ and multijet production. The observed (expected) upper limit on $h h \rightarrow b \bar{b} \tau^{+} \tau^{-}$production is 508 (420) fb which is about 200 (170) times the SM prediction.

\section{Conclusions}

Following the Higgs boson discovery in 2012, the focus has been on measuring the properties of this Higgs boson. All measurements using the LHC Run 1 data are consistent with the SM expectation. Also the analysis of the newly taken LHC Run 2 data shows so far good agreement 
with the SM. The still to be analysed larger Run 2 dataset of approximately $36 \mathrm{fb}^{-1}$ for 2016 and the expected total dataset of $100-150 \mathrm{fb}^{-1}$ until 2018 will improve the measurement precision and offer the possibility to detect yet not-measured Higgs boson production and decay channels. With this total integrated luminosity the measurements will still be limited by the statistical uncertainties with exceptions in the $t t H$ production and $b b$ decays that have expected larger systematic uncertainties $[28,29]$.

\section{References}

[1] F. Englert and R. Brout, Phys. Rev. Lett. 13 (1964) 321-323.

[2] P. W. Higgs, Phys. Rev. Lett. 13 (1964) 508-509.

[3] G. Guralnik,C. Hagen, and T. Kibble, Phys. Rev. Lett. 13 (1964) 585-587.

[4] ATLAS Collaboration, "The ATLAS Experiment at the CERN Large Hadron Collider," JINST 3 (2008) S08003.

[5] CMS Collaboration, “The CMS experiment at the CERN LHC," JINST 3 (2008) S08004.

[6] ATLAS Collaboration, Phys. Lett. B716 (2012) 1-29.

[7] CMS Collaboration, Phys. Lett. B716 (2012) 30-61.

[8] G. Aad et al. [ATLAS and CMS Collaborations], Phys. Rev. Lett. 114 (2015) 191803.

[9] G. Aad et al. [ATLAS and CMS Collaborations], JHEP 1608 (2016) 045.

[10] ATLAS Collaboration, ATLAS-CONF-2016-067, http://cds.cern.ch/record/2206210

[11] CMS Collaboration, CMS-PAS-HIG-16-020, https://cds.cern.ch/record/2205275

[12] ATLAS Collaboration, ATLAS-CONF-2016-079, http://cdsweb.cern.ch/record/2206253

[13] CMS Collaboration, CMS-PAS-HIG-16-033, https://cds.cern.ch/record/2204926

[14] ATLAS Collaboration, ATLAS-CONF-2016-081, http://cdsweb.cern.ch/record/2206272

[15] ATLAS Collaboration, ATLAS-CONF-2016-080, http://cdsweb.cern.ch/record/2206255

[16] CMS Collaboration, CMS-PAS-HIG-16-004, https://cds.cern.ch/record/2139578

[17] ATLAS Collaboration, ATLAS-CONF-2016-058, http://cdsweb.cern.ch/record/2206153

[18] CMS Collaboration, CMS-PAS-HIG-16-022, https://cds.cern.ch/record/2205282

[19] ATLAS Collaboration, ATLAS-CONF-2016-068, http://cdsweb.cern.ch/record/2206211

[20] CMS Collaboration, CMS-PAS-HIG-16-019, https://cds.cern.ch/record/2204925

[21] ATLAS Collaboration, ATLAS-CONF-2016-091, http://cdsweb.cern.ch/record/2206813

[22] ATLAS Collaboration, ATLAS-CONF-2016-063, http://cdsweb.cern.ch/record/2206201

[23] CMS Collaboration, CMS-PAS-HIG-16-003, https://cds.cern.ch/record/2160154

[24] ATLAS Collaboration, ATLAS-CONF-2016-041, http://cdsweb.cern.ch/record/2206079

[25] CMS Collaboration, Phys. Lett. B 744 (2015).

[26] ATLAS Collaboration, ATLAS-CONF-2016-049, http://cdsweb.cern.ch/record/2206131

[27] CMS Collaboration, CMS-PAS-HIG-16-028, https://cds.cern.ch/record/2204934

[28] ATLAS Collaboration, ATL-PHYS-PUB-2014-016, https://cds.cern.ch/record/1956710

[29] CMS Collaboration, CMS-NOTE-2013-002, https://arxiv.org/abs/1307.7135 